\title{
Differenz
}

Revista internacional de estudios heideggerianos y sus derivas contemporáneas

AÑO 8, NÚMERO 7: JULIO DE 2021. ISSN 2695-9011 - e-ISSN 2386-4877 - DOI: 10.12795/Differenz.2021.i07.06 [pp. 121-125]

Recibido: 16/03/2021

Aceptado: 07/05/2021

\section{BASSO MONTEVERDE, Leticia (Ed.) (2020). Heidegger y su obra. Ensayos en torno a la unidad de su pensar. Buenos Aires: Biblos. 256 pp.}

\author{
Esteban Raúl Cardone \\ Universidad Nacional de Mar del Plata-Universidad Nacional de Lanús
}

Resulta sabido que aproximarse al pensamiento de Martin Heidegger constituye una experiencia ardua. Valdría mencionar al menos tres razones entre las dificultades que se asocian con su lectura. En primer lugar hay que sortear la dificultad linguística asociada a su forma de enfrentarse a la tradición filosófica. En segundo término, el núcleo fundamental que estructura toda su obra, la pregunta que interroga por el sentido del ser, suele causar extrañeza, incomprensión e incluso reticencia. Finalmente, dado que el proyecto heideggeriano es el fruto de una reflexión madura acerca de la tradición filosófica que lo precede, exige al lector ciertas destrezas ya asimiladas en el campo de la filosofía. Por fortuna, existe abundante bibliografía capaz de asistirnos a remediar nuestras carencias. Al respecto, la Introducción a Heidegger de Gianni Vattimo suele encontrarse entre la literatura de carácter propedéutico más citada. Podemos agregar también el trabajo de autores como Ramón Rodriguez, Jesús Adrián Escudero, Dina Picotti y Rüdiger Safranski 
como oportunidades valiosas para acercarse a la obra del filósofo alemán ${ }^{1}$. Heidegger y su obra: ensayos sobre la unidad de su pensar no es un texto introductorio al pensamiento del autor, sino un ejemplo del esfuerzo conjunto de estudiosos de la obra heideggeriana puestos a problematizar su legado filosófico, a ampliar las derivas de un pensamiento aplicado a órdenes tan diversos como el arte y la técnica, entre otros. Los artículos que forman la compilación son el producto de un trabajo erudito, aplicado mayormente a problemas intra teóricos específicos.

La unidad de la obra heideggeriana comporta por sí mismo un problema dado que se encuentra atravesada por un denominado viraje en su perspectiva, percibido por el propio filósofo alemán y hecho explícito en la Carta sobre el humanismo del año 1947.¿cómo entender un pensamiento que declara su propia insuficiencia? ¿No comporta el fracaso una ganancia necesaria de toda deriva posterior? Los estudios que componen el título que edita Leticia Basso Monteverde ${ }^{2}$ se dirigen a atender la correspondencia y unidad entre los momentos diversos del itinerario filosófico de Heidegger. En tal sentido, la propuesta del texto acierta al ofrecer una lectura unitaria de la obra del filósofo alemán por sobre aquellas fragmentarias que tanto énfasis le otorgan a la disección tajante de momentos y pierden de vista la integridad del proyecto en su totalidad. Después de todo, la pregunta por el ser es la constante de toda su prólifica trayectoria filosófica.

Las seis investigaciones que componen el texto no exigen ningún orden de lectura; cada una se dedica a una problemática o ámbito específico. De modo que lo que aquí se distingue se suscribe a la preferencia provisional de quien releva el texto.

En el artículo que inaugura el recorrido se pregunta Adrián Bertorello cómo leer a Heidegger, a sabiendas de que no existe un modo definitivo de hacerlo, dado que todo encuentro con un texto comporta un choque de participaciones signada por apropiaciones, lecturas y entredichos que hacen a la riqueza propia de la situación fáctica de enunciación.

1 Los autores mencionados se ofrecen al lector apenas como una coordenada de aproximación a la obra de Martin Heidegger. De Rüdiger Safranski puede consultarse su obra Un maestro de alemania. Martin Heidegger y su tiempo, publicado en español por Tusquets editores. Editorial Herder publica en dos tomos la muy útil Guía de lectura de Ser y Tiempo de Martin Heidegger, de Jesús Adrián Escudero. Merece mención el trabajo que Ramón Rodriguez dedica al filósofo alemán tanto en Heidegger y la crisis de la época moderna como en La transformación hermenéutica de la fenomenología. Una interpretación de la obra temprana de Heidegger. Finalmente, el texto Heidegger: una introducción de Dina Picotti resulta un aporte valioso de acercamiento al pensamiento del autor.

2 Leticia Basso Monteverde, quien realiza la compilación, es doctora en filosofía por la Universidad de Buenos Aires e investigadora adjunta de Conicet. Trabaja además como docente en la Universidad Nacional de Mar del Plata (Argentina). Investigadora en la Agencia Nacional de Investigación Científica y Tecnológica y miembro de la Sociedad Iberoamericana de Estudios Heideggerianos. 
Bertorello hace explícitas las condiciones inherentes al encuentro entre autor y lector. Así, la recepción de un texto se entiende como un vínculo que no se resuelve simplemente al aproximarse a lo disponible en él sino al actualizar su potencial contenido. El artículo de Bertorello es en cierto modo profético, nos anticipa un problema que está presente en el resto de las producciones que lo acompañan. La pregunta sobre cómo es posible leer El origen de la obra de arte (1936) encuentra respuesta a partir de dos posibilidades, según se lleve adelante un régimen de lectura respetuoso de la interpretación del propio autor u otro capaz de entrecruzar lo dado con otros conceptos e incluso disciplinas. El primer modo de lectura evidencia las decisiones interpretativas del propio Heidegger. Como consecuencia la reflexión en torno al arte se comprende dentro del proyecto más amplio que contempla el sentido de la pregunta por el ser. El segundo modo de acercamiento al texto arriesga una lectura que lo entrecruza con el ámbito de la semiótica; la pregunta por el ser nos ordena a hablar del sentido, habiendo una raíz común entre ontología y espacio semiótico. De este modo, una lectura en clave semiótica de El origen de la obra de arte es capaz de advertir en el texto las condiciones de producción de sentido que inaugura la obra de arte como dispositivo semiótico. No es difícil encontrar menciones en torno a la decepción que el propio Heidegger sufrió con la recepción de Ser y Tiempo. A decir verdad, toda su trayectoria filosófica estuvo signada por la polémica con sus lectores, a quienes el filósofo no dudaba en reprochar falta de comprensión. Podría pensarse entonces que Heidegger abogaba por ser complacido con una lectura propia, capaz de ceñirse a sus indicaciones interpretativas, de hablar el lenguaje del autor y con ello de estar a la altura de lo que se propone. Sin embargo su obra abunda, incluso abusa a veces, de lecturas que tuercen el sentido de los términos o fuerza interpretaciones, dando como resultado algunos de los momentos más lúcidos y originales de la historia del pensamiento más reciente.

A propósito de cómo la recepción de Ser y Tiempo condicionó la posterior elaboración filosófica de Heidegger se extiende Francois Jaran, quien se dispone a esclarecer cuestiones concernientes a los motivos del inacabamiento de su obra magna, en base al decir del propio autor en los Cuadernos negros. Las "insuficiencias del lenguaje" denunciadas por el propio Heidegger como motivo del "fracaso" del camino que inauguró Ser y Tiempo, serían la prueba de una dificultad que no habría podido sortear: la ruptura adecuada con la tradición filosófica. A decir verdad, Heidegger mismo reconocería que Ser y Tiempo adeudaba demasiado para su gusto de corrientes filosóficas como el neokantismo, la fenomenología y el existencialismo de Kieerkegard. Paradójicamente interpretaría como requisito de inteligibilidad de su propia obra el requerir cierto grado de determinación de la tradición que pretende impugnar, lo cual constituye el reconocimiento de que sin un 
haber previo no habría lector posible. La recepción de Ser y Tiempo aún hoy ha quedado envuelta en interpretaciones de carácter antropológico o existencialista, que la recortan del proyecto filosófico general del autor. Al respecto, varias expresiones de Heidegger demuestran ingratitud para con sus lectores. El silencio, concluiría Heidegger, sería el remedio a la incomprensión ruidosa. La demostrada erudición de Heidegger no lo vuelve un autor solícito al debate con sus contemporáneos. El artículo busca demostrar que la frustrada recepción de Ser y Tiempo constituye un ingrediente necesario para comprender su inacabamiento y a la vez su vigencia.

La problematicidad inherente a la producción y recepción del discurso no deja de estar presente en el artículo de Luciano Mascaró, quien se ocupa del devenir del pensamiento heideggeriano acerca de la técnica. Cuán significativo resulta el discurrir filosófico de Heidegger acerca de la técnica es la pregunta que moviliza la reflexión del autor, quien se esfuerza por demostrar que el pensamiento temprano del filósofo alemán resulta más rico al ámbito de la filosofía de la técnica que lo dicho en la conferencia de 1953 titulada La pregunta por la técnica. Al respecto señala Mascaró que en Ser y Tiempo el útil es el ente sobre el cual recae nuestro trato más inmediato con el mundo, entendido éste último como una enorme trama de sentidos pragmáticos. Así, el comparecer del mundo se oculta ante el servicio efectivo de los entes. El detenimiento filosófico en el infortunio práctico es, a juicio de Mascaró, el mayor aporte de Heidegger a la filosofía de la técnica, quien descubre en la inoperancia y en la inutilidad una modalidad de experiencia del mundo. El fracaso del útil conduce a que nuestra atención se detenga en él, constatando en tal simple hecho el plexo de sentidos en el que se inserta el proyecto del Dasein. El artefacto técnico contribuye a configurar el espacio antes que cualquier abordaje de la ciencia en particular. De allí que el autor sostenga que "la experiencia originaria del espacio aparece en la misma interacción del trato técnico en el mundo", deshabilitando así una concepción del espacio como índice previo de toda instalación humana. No obstante los caminos que sugirió el pensamiento heideggeriano no alcanzaron un nivel de actualización acorde con los desarrollos técnicos de su época, dejando truncas infinidad de líneas de análisis. La reflexión posterior a Ser y Tiempo, puntualmente la que se congrega en la conferencia del año 1953, se ocupa de la esencia de la técnica como forma particular de desocultamiento atento al cálculo, la disponibilidad y el dominio del ente. Lejos de convertirse en una diatriba apocalíptica en contra de la técnica cifra también expectativas provechosas en dicho modo de vínculo con el mundo.

Los autores que participan del volumen publicado por Editorial Biblos poseen una amplia trayectoria de estudio de la obra de Martin Heidegger, que los ubica como miembros de la Sociedad Iberoamericana de Estudios Heideggerianos y de renombrados 
organismos de investigación científico-filosófica alrededor del mundo. Al orden de sus incumbencias se suma además el desempeño en diversas universidades tanto de Argentina como de Canada, España y México, que respaldan con una formación dedicada a la filosofía en su mayor nivel académico. Ello le garantiza a los artículos aquí reunidos un nivel de profundidad acorde a la calidad de la discusión filosófica contemporánea. Al trabajo reseñado de Adrian Bertorello, Francois Jarán y Luciano Mascaró, se agrega la participación de Alejandro Vigo, Roberto Walton y Ángel Xolocotzi Yáñez.

Heidegger y su obra: ensayos en torno a la unidad de su pensar resulta una cita de encuentro propicia con las derivas actuales del pensamiento heideggeriano siendo ejemplo de una reflexión filosófica madura en torno a la obra del filósofo alemán. La compilación que realiza Leticia Basso Monteverde ofrece una buena oportunidad para comprender la obra de Heidegger en la unidad de su trayectoria, evitando con ello la visión que parcializa la consideración de su itinerario y que concentra su atención en su obra más renombrada, Ser y Tiempo, sin atender a su proyecto filosófico de forma integral. Con ello los autores incluidos en la compilación nos invitan a considerar una pauta algo más general del ejercicio de la filosofía, a saber, que al pensar acuden vacilaciones como avances, direcciones y tropiezos; en definitiva, que la filosofía se nutre de la inquietud mucho más que de las certezas. 